\title{
Intestinal inflammation and activation of sensory nerve pathways: a functional and morphological study in the nematode infected rat
}

R De Giorgio, G Barbara, P Blennerhassett, L Wang, V Stanghellini, R Corinaldesi, S M Collins, G Tougas

\begin{abstract}
Background-In the rat, gastric distension elicits an intensity dependent pseudoaffective bradycardia mediated via capsaicin sensitive afferent and cholinergic efferent vagal pathways. Inflammation alters visceral perception although the mediators responsible have not been identified. In the nematode infected rat, there is a substantial increase in neuronal substance $P(S P)$ content of the gut.

Aims-To examine the effects of inflammation on perception of a noxious visceral stimulus and on $S P$ and neurokinin 1 (NK-1) receptor immunoreactivity (IR) in visceral afferent pathways.

Methods-Immunohistochemistry was performed on sections from the jejunum, dorsal root ganglia (DRG), and spinal cord (T1-L1) using SP and NK-1 rabbit polyclonal antibodies. In the DRG, the number of SP-IR or NK-1-IR neurones per section was visually quantified. The pseudoaffective cardiac reflex response to gastric stimulation was compared in control and Trichinella spiralis infected rats.
\end{abstract}

Results-Intestinal inflammation induced a rightward shift in the intensity dependent bradycardic response to gastric distension. This was associated with a marked increase in SP-IR not only in the gut wall but also in the DRG and dorsal horn of the spine. In contrast, NK-1-IR was not increased in the gut wall. Moreover, inflammation evoked a decrease in NK-1-IR in the dorsal horn. No NK-1-IR was identified in the DRG of either control or infected animals.

Conclusions-Intestinal inflammation modulates the capsaicin sensitive pseudoaffective autonomic response to gastric distension, increases SP-IR in afferent pathways, and downregulates dorsal horn NK-1-IR. As the pseudoaffective response is capsaicin sensitive, the rightward shift of the response is likely the consequence of the decrease in NK-1 receptors in the sensory pathways.

(Gut 2001;49:822-827)

Keywords: tachykinin peptides; neurokinin receptors; sensory afferent neurones; intestinal inflammation; neuroimmune interactions; nematode infection; pseudoaffective response
Noxious gastric distension produces a pseudoaffective cardioautonomic response characterised by bradycardia which is dependent on the distension volume. ${ }^{12}$ This effect depends predominantly on activation of vagal afferent pathways as well as on an atropine sensitive cholinergic efferent pathway. Furthermore, the pseudoaffective cardioautonomic response to gastric distension can be abolished by neonatal or perivagal capsaicin thus implicating a capsaicin sensitive vagal afferent pathway, almost certainly involving the tachykinin peptide substance $\mathrm{P}(\mathrm{SP})$, as well as other sensory neuropeptides. ${ }^{3}$

There is clear evidence indicating extensive spinal convergence of stimuli arising from different visceral organs. ${ }^{4}$ A typical example of viscerovisceral convergence to the second order neurone within the dorsal horn of the spinal cord is that produced by gall bladder distension which evokes angina-like chest pain. ${ }^{4}$

The sensory response to inflammation of the gastrointestinal tract has traditionally been studied in an acute setting following local application of several mucosal irritant substances such as turpentine. ${ }^{67}$ These studies have been restricted to examining sensory afferent pathways and have not incorporated an assessment of the broad autonomic response that has long been considered an important component of sensory activation by noxious stimuli. $^{8}$

The pseudoaffective response to noxious gastric distension is mediated primarily by vagal sensory nerves, ${ }^{12}$ likely containing the neuropeptide SP. Previously, we demonstrated that the primary infection of rats with the nematode parasite Trichinella spiralis caused an eightfold increase in immunoreactive SP in the proximal gut. ${ }^{9}$ SP was depleted by scorpion venom or by capsaicin treatment, thus demonstrating its location in capsaicin sensitive nerves. The increase in SP was prevented by steroid treatment of the rats during infection, and was not observed in infected athymic rats, indicating that the change in this important sensory neuropeptide occurred as a result of the inflammatory and immune response to infection, and that $\mathrm{T}$ lymphocytes were critical. Further studies in this model indicated that infection was accompanied by a substantial downregulation of the neuropeptide degrading enzyme neutral endopeptidase in the gut,

Abbreviations used in this paper: DRG, dorsal root ganglia; IR, immunoreactive/immunoreactivity; NK, neurokinin; SP, substance P. 
whose decrease would serve to greatly increase the bioavailability of SP released following stimulation of sensory nerves in the inflamed gut. ${ }^{10}$

In the present study, we examined the impact of inflammation induced by $T$ spiralis infection on pseudoaffective response. Given the wide convergence of both vagal and splanchnic sensory fibres onto second order neurones at the spinal level, we elected to measure cardiac autonomic responses following gastric distension in the rat. ${ }^{45}$ Specifically, we measured the capsaicin dependent pseudoaffective cardiac autonomic response, as well as the downstream changes in SP containing spinal afferent neurones. Our results indicated that noxious gastric distension in this model produced a pseudoaffective cardiac autonomic response that was shifted to the right. We also showed that there was a significant increase in $\mathrm{SP}$, but not neurokinin 1 (NK-1, the preferred receptor for SP) immunoreactive (IR) fibres in the mucosa and muscle layers, and an increase in the number of SP-IR dorsal root ganglion (DRG) neurones in infected rats compared with controls. However, in the dorsal horn of the spinal cord of $T$ spiralis infected rats, along with an increase in SP-IR nerves, there was a marked compensatory decrease in NK-1 receptor expression.

\section{Materials and methods}

ANIMALS AND T SPIRALIS INFECTION

Male Sprague-Dawley rats (200-250 g) (purchased from Charles River Laboratories) were kept in filtered cages under controlled ambient temperature and light-dark cycle (14:10 hours). Rats were infected by administering 1.0 $\mathrm{ml}$ of phosphate buffered saline containing $7500 T$ spiralis larvae by gavage. The larvae were obtained from male CD1 mice infected 30-90 days previously by $T$ spiralis using a previously described method. ${ }^{11}$ Animals had unrestrained access to water but were fasted for 18 hours before experiments. Rats were studied six days after infection and age matched non-infected animals served as controls. For immunohistochemistry, animals were killed by cervical dislocation. For functional studies, rats were anaesthetised with a ketamine hydrochloride $(90 \mathrm{mg} / \mathrm{kg})$ and xylazine $(20 \mathrm{mg} / \mathrm{kg})$ mixture given intramuscularly and were placed on a heating pad to maintain the animal's temperature at about $36^{\circ} \mathrm{C}$ throughout the duration of the study. Supplemental anaesthesia was given throughout the study as required. At the end of the pseudoaffective response experiment (see below), animals were killed by cervical dislocation. All experimental procedures were approved by the Animal Care Committee at McMaster University and were in accordance with guidelines of the Canadian Council on Animal Care.

PSEUDOAFFECTIVE CARDIOAUTONOMIC RESPONSE TO GASTRIC DISTENSION

The approach has been described in detail previously, including the afferent and efferent pathways involved. ${ }^{2}$ Briefly, the cardioautonomic reflex response to noxious gastric distension obtained in control animals was compared with the response observed in age, sex, and weight matched $T$ spiralis infected rats ( $n=6$ in both groups). Following laparotomy and duodenotomy, a $2 \times 2 \mathrm{~cm}$ balloon was inserted into the duodenum and subsequently positioned into the stomach. The gastric volume-cardiac response relationship was then examined using volumes ranging from 3 to 15 $\mathrm{ml}$, which were applied for a 60 second period. The response to each volume was studied on three separate occasions in each animal. Continuous electrocardiographic recordings were performed through three electrodes applied on the right and left shoulders and the left leg of the animal.

\section{IMMUNOHISTOCHEMISTRY}

Specimens of jejunum, DRG (T1-L1), and spinal cord obtained from $T$ spiralis infected and control rats ( $n=6$ each group) were rapidly removed and processed for either routine histology or immunohistochemistry. For the latter, tissues were fixed by immersion in $4 \%$ paraformaldehyde in $0.1 \mathrm{M}$ phosphate buffer, pH 7.4, for $6-8$ hours at $4^{\circ} \mathrm{C}$ and subsequently placed in $25 \%$ sucrose in $0.1 \mathrm{M}$ phosphate buffer for cryoprotection until sectioning. Specimens were cut with a cryostat at 10-12 $\mu \mathrm{m}$, mounted onto chrome-alum gelatin coated slides, and processed with the immunofluorescence method, as previously described. ${ }^{12}$ The antibodies used included a rabbit polyclonal antibody anti-SP, directed against the $\mathrm{C}$ terminal portion of the tachykinin amino acid sequence (antibody code 8701, diluted $1: 500),{ }^{12}{ }^{13}$ and a rabbit polyclonal anti-NK-1, directed against the $\mathrm{C}$ terminus of the intracellular loop of the receptor sequence (diluted 1:1000). ${ }^{14}$

Specificity studies included control experiments commonly applied in immunohistochemical protocols. ${ }^{12-14}$

Sections were analysed by a Leitz Orthoplan microscope equipped with a Ploem epiillumination system with "I2" or "L2" filter cubes to detect fluorescence.

IMMUNOREACTIVE PATTERNS AND QUANTITATIVE ANALYSIS OF SP IMMUNOLABELLED GANGLION CELL BODIES

Tissue sections from both infected and controls rats were processed for immunohistochemistry simultaneously. Comparison between infected and control sections was performed in parallel by two examiners (RDeG and $\mathrm{GB}$ ) in a blinded fashion.

The number (mean (SEM)) of immunolabelled SP neural cell bodies per section in each DRG (from T1 to L1) was evaluated by counting the labelled perikarya by means of direct microscopic analysis. This analysis was performed on 12 consecutive sections per each DRG of infected and control rats.

STATISTICAL ANALYSIS

All studies involved at least four rats. For quantitative analysis of SP labelled DRG neural cells, the Mann-Whitney test for unpaired data was used. Change in heart rate was expressed 


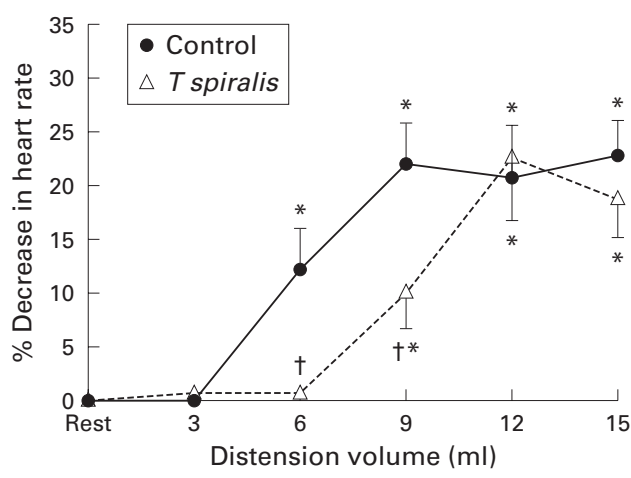

Figure 1 Volume dependent pseudoaffective increase in heart rate associated with gastric distension in control and Trichinella spiralis infected rats. Data are presented as mean (SD) per cent decrease in heart rate compared with resting heart rate immediately before distension. ${ }^{\star} p<0.05$ versus resting heart rate; $t p<0.05$ versus control animals.

as per cent change in heart rate compared with the immediately preceding 60 second control period before distension. The Student's $t$ test and ANOVA (one and two way, and repeated measures) were used as appropriate. Statistical significance was determined at $\mathrm{p}<0.05$.
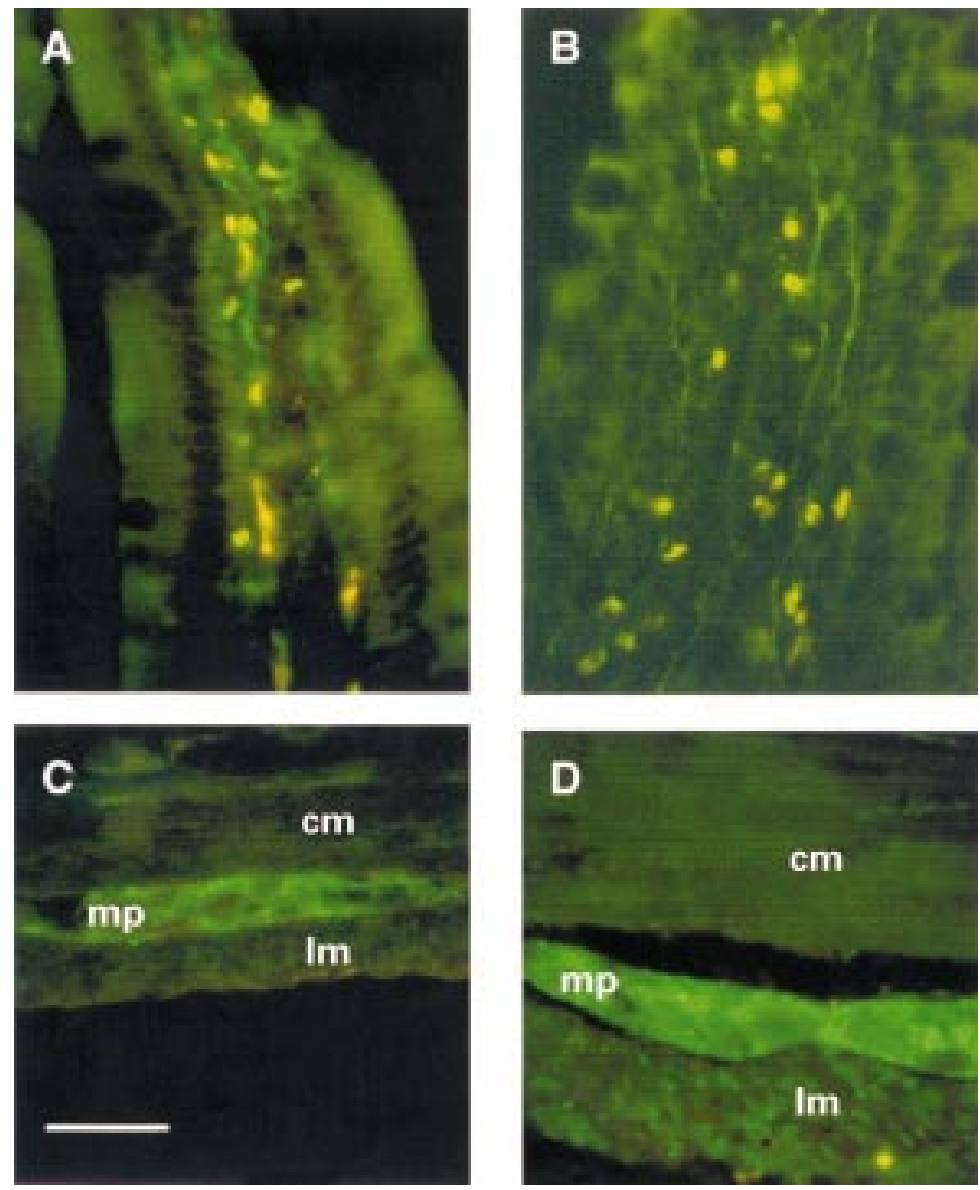

Figure 2 Representative photomicrographs showing substance P (SP) immunoreactivity in the control $(A, C)$ and Trichinella spiralis infected $(B, D)$ jejunum. Note the marked increase in the density and staining intensity of the SP-immunoreactive (IR) nerve processes running throughout the villi in the inflamed jejunum (B) in comparison with controls $(A)$. An increase in SP-IR was also found in myenteric neurones of the inflamed jejunum (D) in comparison with controls (C). cm, circular muscle; lm, longitudinal muscle; mp, myenteric plexus. Calibration bar, $25 \mu \mathrm{m}$.

\section{Results}

PSEUDOAFFECTIVE CARDIAC RESPONSE TO

GASTRIC DISTENSION

In control animals, as previously documented, gastric distension elicited a volume dependent pseudoaffective decrease in heart rate which was first present with volumes of $6 \mathrm{ml}$ and maximal with volumes ranging from 9 to $15 \mathrm{ml}$ $(\mathrm{p}<0.05 v$ resting heart rate). $T$ spiralis infection did not change resting intraballoon pressures. In $T$ spiralis infected animals (fig 1 ), there also was a similar volume dependent pseudoaffective cardioautonomic reflex response but while the magnitude of the response was comparable, there was a significant rightward shift in $T$ spiralis infected animals.

\section{JEJUNUM}

Specificity was demonstrated by lack of immunoreaction in sections in which the primary antisera preadsorbed with the appropriate peptides.

In the jejunum of control rats, SP-IR (fig 2) was identified in varicose nerve endings targeting the different layers of the gut wall as well as in myenteric and submucosal ganglion cell bodies. In particular, nerve processes positive for SP-IR supplied the muscularis externa, the submucosal vasculature, and the mucosa (fig 2A). A rather dense network of SP labelled processes was found in the deep muscular plexus, the lining situated between the inner part of the muscle layer, and the submucosa of the jejunum. SP containing nerve fibres were also identified around stained and unstained ganglion cells of the submucosal and, to a lower extent, myenteric plexus (fig 2C). NK-1-IR (fig 3 ) was identified mainly in myenteric and submucosal neurones of the rat jejunum as well as in nerve fibres occasionally identified either within the myenteric plexus or distributed in the muscular layer. No NK-1 immunolabelling was identified around blood vessels in the submucosa or in the mucosa of the jejunum. However, in the deep muscular plexus of the jejunum, NK-1 staining was localised to non-neuronal cells whose appearance was that of the interstitial cells of Cajal (fig 3A).

The distribution pattern of both SP and NK-1-IRs was not modified in $T$ spiralis infected rats. However, in infected rats, SP containing nerves showed an increased number and staining intensity in the muscularis externa and mucosa (fig 2B) compared with controls (fig 2A). Also, SP-IR was markedly increased in submucosal and myenteric neurones (fig 2D) in the small intestine of infected animals in comparison with controls (fig 2C). In contrast, NK-1-IR displayed a reduction in staining intensity both in enteric neurones (that is, myenteric and submucosal plexus) and particularly in the interstitial cells of Cajal (fig 3B) compared with controls (fig 3A).

DRG

SP immunolabelling was localised to small and medium sized neuronal cell bodies and nerves from $\mathrm{T} 1$ to $\mathrm{L} 1$ of control animals (fig 4A). In infected animals, SP-IR cell bodies showed increased density and staining intensity (fig 

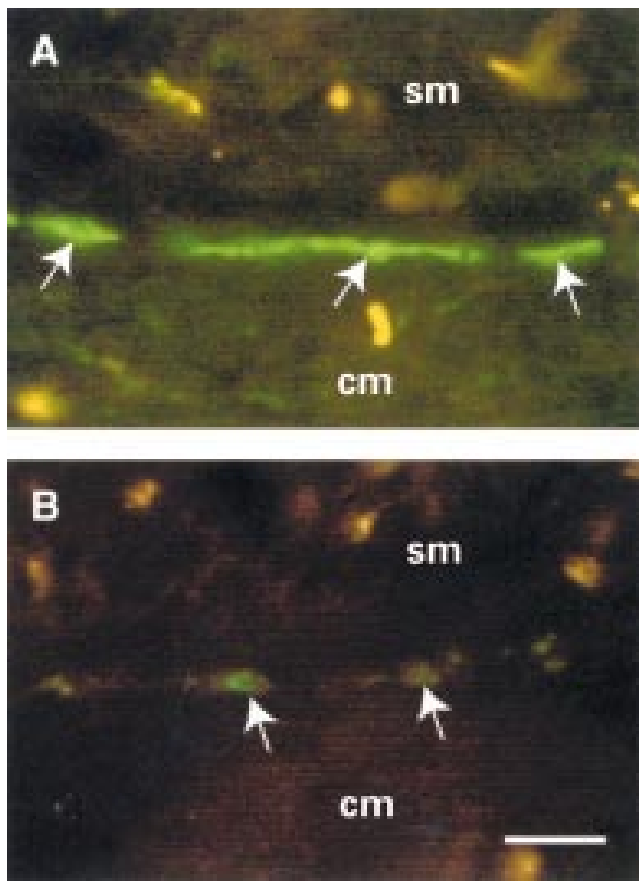

Figure 3 Neurokinin 1-immunoreactivity (NK-1-IR) in the jejunal deep muscular plexus of Trichinella spiralis infected (B) and control (A) rats. NK-1 receptor immunolabelling was identified in non-neuronal elements with the appearance of interstitial cells of Cajal (arrows in A). In inflamed jejunum (B), NK-1 immunostaining localised to the interstitial cells of Cajal (arrows) was markedly decreased in comparison with controls. cm, circular muscle; sm, submucosa. Calibration bar, $25 \mu m$.

4B). As shown in fig 5, these findings were confirmed by quantitative analysis which demonstrated a significantly increased number of SP containing neurones in infected compared
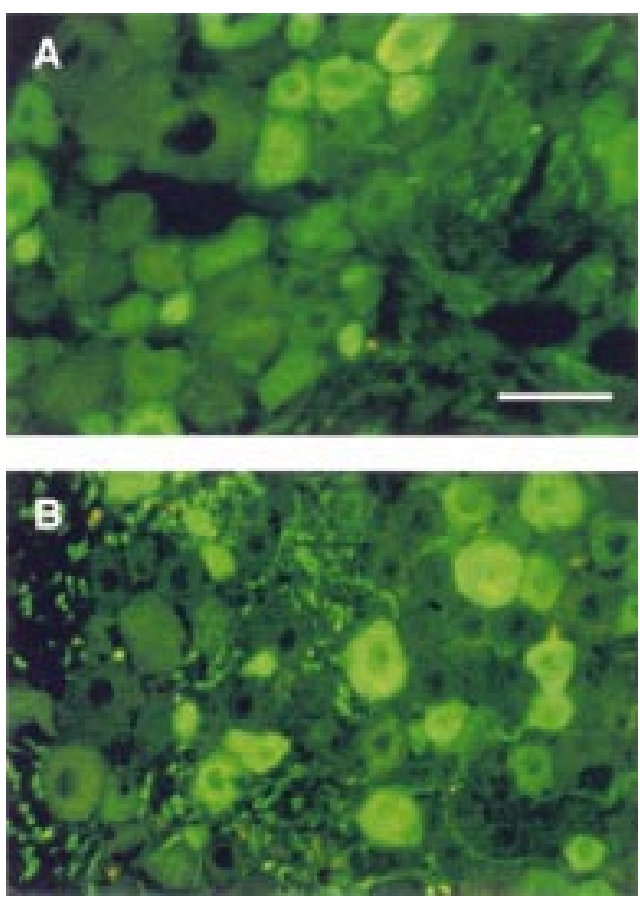

Figure 4 Photomicrographs showing substance $P$-immunoreactive (SP-IR) neurones of the dorsal root ganglia (DRG) in Trichinella spiralis infected $(B)$ and control (A) rats. Note the increased SP-IR in the small-medium sized DRG neurones of T spiralis infected (B) in comparison with controls $(A)$. Calibration bar, $25 \mu \mathrm{m}$.

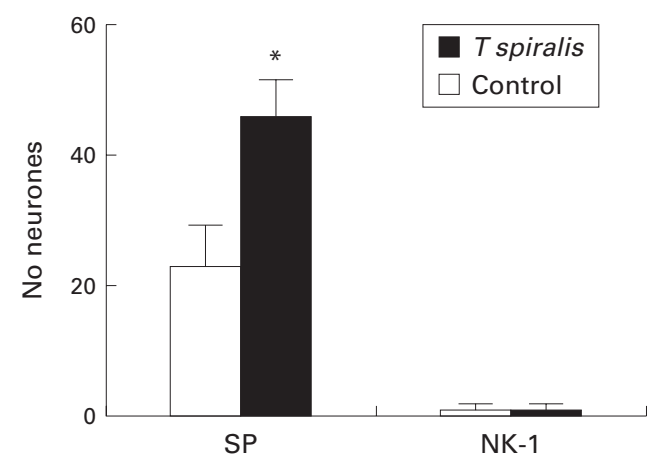

Figure 5 Mean (SEM) number of substance $P$-immunoreactivity (SP-IR) dorsal root ganglia (DRG) neurones in the Trichinella spiralis infected and control rats. Neurokinin 1 (NK-1) is also shown, although this tachykinin receptor was not detected in any ganglion cells of either infected or control rats. ${ }^{\star} p<0.05$ versus controls.

with control rats $(57$ (8) $v 23$ (7) positive cell bodies, respectively; $\mathrm{p}<0.05$ ). No NK-1-IR ganglion cell bodies were observed in any infected or control rats.

\section{SPINAL CORD}

In the spinal cord of control rats, SP (fig 6A, B) and to a minor extent NK-1 (fig 6C, D) labelled nerve processes were seen predominantly in the outermost laminae of the dorsal horn. Conversely, in infected rats, there was a marked increase in the density and staining intensity of SP containing nerves in the dorsal horn (fig 6B) whereas NK-1-IR was undetectable (fig 6D).

\section{Discussion}

In the present study, we have used the pseudoaffective cardioautonomic response to gastric distension as an index of the intensity of the autonomic reflex response to a noxious visceral stimulus. This response is primarily mediated through capsaicin sensitive vagal and splanchnic (sympathetic) visceral afferent pathways. We have recently shown that distension of the rectum also elicits a similar volume dependent reflex bradycardia in rats, although the primary afferents involved are exclusively sympathetic. ${ }^{15}$

Neonatal capsaicin prevents the pseudoaffective cardioautonomic response to either gastric or colonic distension, ${ }^{216}$ indicating the essential role of SP in this response. In the present study, we observed a rightward shift in the pseudoaffective response to gastric distension in $T$ spiralis infected animals. We interpret this finding as a consequence of altered SP content expression in dorsal horn neural networks.

The role exerted by SP on the perception of potentially noxious visceral stimuli in the present model of intestinal inflammation is also likely related to modulation of NK-1, and possibly NK-2 and NK-3, tachykinin receptors in the spinal cord. This possibility has previously been raised by others who have suggested a role for tachykinin receptors (particularly NK-2 and NK-3 receptors) in the altered visceral perception associated with zymosan colitis in the rat colon. ${ }^{17}$ The present results suggest that 

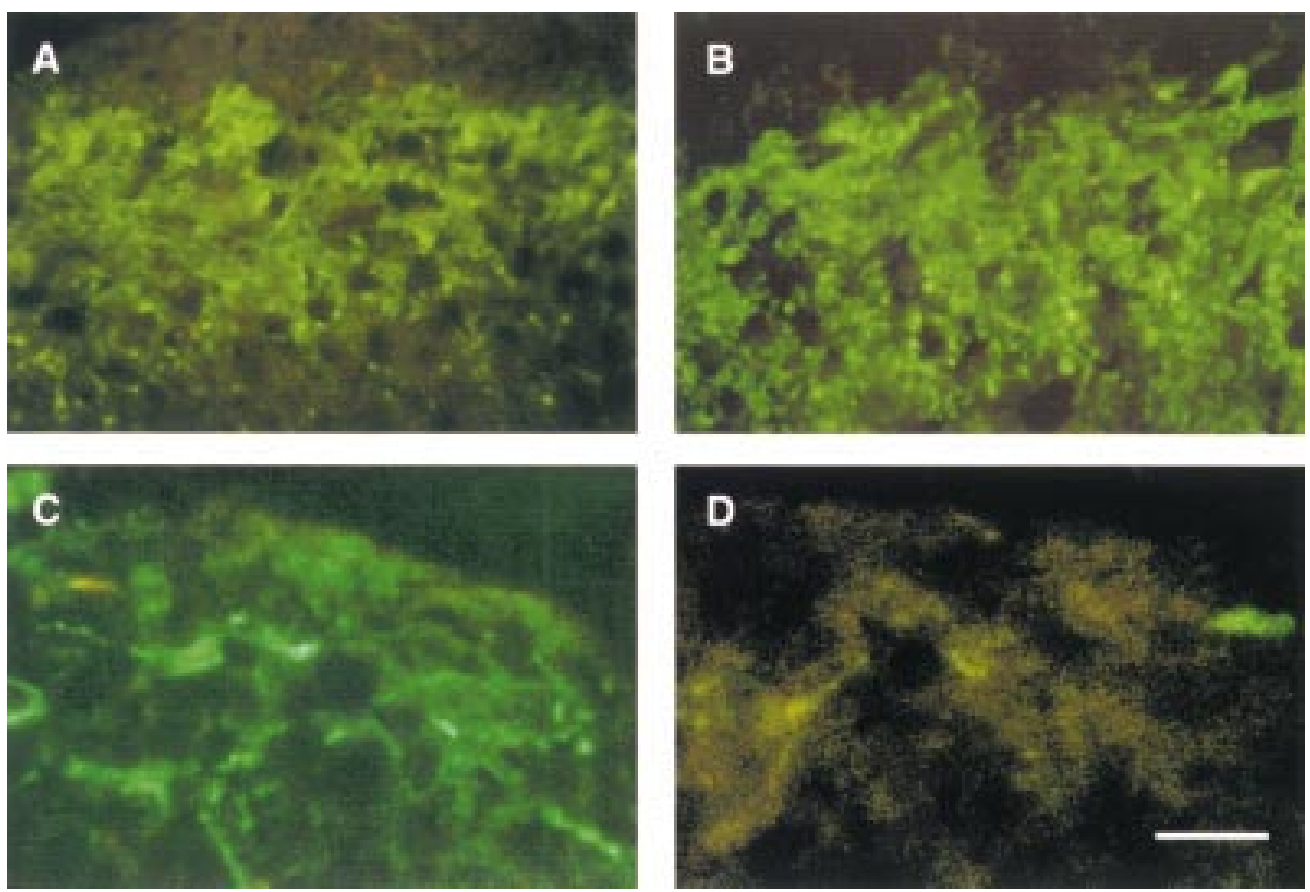

Figure 6 Representative photomicrographs showing substance $P$-immunoreactivity (SP-IR) $(A, B)$ and neurokinin 1 -immunoreactivity $(N K-1-I R)(C, D)$ in the spinal cord of control $(A, C)$ and Trichinella spiralis infected $(B, D)$ rats. In the spinal cord of control animals, SP immunostaining was detectable in nerve fibres $(A)$ whereas NK-1-IR was visualised in neurones and nerve fibres $(C)$ mainly identified in the outermost laminae of the dorsal horns. In $T$ spiralis infected rats, there was a striking increase in the density and staining intensity of SP neural network (B) compared with controls (A). In relation to the SP increase, we found a marked decrease in NK-1-IR in the dorsal horn of T spiralis infected rats (D) compared with controls (C). Calibration bar, $25 \mu \mathrm{m}$.

similar modulation of tachykinin receptor expression may occur in response to nematode induced enteritis.

Inflammatory conditions of the gastrointestinal tract are associated with increased visceral perception and nociception both in human and animal models. ${ }^{6718}$ As it is generally believed that SP plays a crucial role in the transmission of nociceptive sensory stimuli along visceral afferent pathways, we examined SP-IR and NK-1-IR in the DRG. We found a significant increase in SP-IR in DRG cell bodies, consistent with a previous report in Clostridium difficile toxin enteritis in rats. ${ }^{19} \mathrm{We}$ have previously shown attenuation of increased SP-IR in afferent nerve fibres of either steroid treated or athymic $T$ spiralis infected rats, ${ }^{9}$ indicating that it is the immune response rather than the parasite per se that is responsible for SP neuronal changes. Furthermore, cytokines such as interleukin $1 \beta^{20}$ and tumour necrosis factor $\alpha,{ }^{21}$ whose gene expression is enhanced during $T$ spiralis infection, may also contribute to the increase in SP.

Tissue inflammation is known to alter afferent visceral function, leading to hyperalgesia. While SP is recognised as an important sensory neurotransmitter, the precise role of SP and NK-1 receptors in inflammation induced hyperalgesia is poorly understood. Using a specific antibody, we found a distribution of NK-1 receptor in fibres and neurones throughout the dorsal horn of control animals, a finding which confirms previously published data. ${ }^{22-24}$ The increased SP-IR observed in $T$ spiralis infected rats was accompanied by a decrease in NK-1 receptors in nerve fibres and neurones of the dorsal horn. This finding likely reflects internalisation of the NK-1 receptor, a cellular process leading to desensitisation (and thereby to resensitisation), which is relevant to the regulation of neural excitability. ${ }^{25}$ For example, in spinal cord neurones, SP release from sensory endings has been shown to induce NK-1 receptor internalisation. ${ }^{26}{ }^{27}$ In animal models of somatic inflammation, such as that of persistent hindpaw inflammation, it has been recently demonstrated that the inflammatory stimulus alone determines upregulation of NK-1 receptors in dorsal horn neurones. ${ }^{26} 28$ However, in this setting, addition of noxious and non-noxious mechanical or thermal stimulation determined increased internalisation of NK-1 receptors. If a parallel between somatic and visceral sensory pathways exists, then our finding of a marked decrease in NK-1 receptor expression would imply the presence of additional stimuli (that is, mechanical and/or chemical) arising from the inflamed gut. In support of this hypothesis, studies from this and other laboratories have shown profound changes in secretory and motor function in the nematode model of intestinal inflammation. ${ }^{29}$

This work was supported by grants from the Medical Research Council of Canada and by the National Institute of Health (DK52388). The authors wish to thank Ms Helen C Wong, Drs John H Walsh, and Catia Sternini of the Antibody Core subsection of DK 41301 CURE: Digestive Diseases Research Center, UCLA School of Medicine, and Dr Steve Vigna, Duke University Medical Center, Durham, North Carolina, for the generous gift of the anti-SP (8701) and anti-NK-1 receptor antibodies, respectively.

1 Grundy D, Davison JS. Cardiovascular changes elicited by vagal gastric afferents in the rat. $Q \mathcal{F}$ Exp Physiol 1981;66:307-10. 
2 Tougas G, Wang L. Pseudoaffective cardioautonomic responses to gastric distention in rats. Am $\mathcal{f}$ Physiol responses to gas

3 Maggio JE. Tachykinins. Ann Rev Neurosci 1988;11:13-21.

4 Ness TJ, Gebhart GF. Visceral pain: a review of experimental studies. Pain 1990;41:167-234.

5 Foreman RD. Organization of spinothalamic tract as a relay for cardiopulmonary sympathetic afferent fiber activity. Prog Sens Physiol 1989;9:1-51.

6 Mayer EA, Gebhart GF. Basic and clinical aspects of visceral hyperalgesia. Gastroenterology 1994;107:271-93.

7 Bueno L, Fioramonti J, Delvaux M, et al. Mediators and pharmacology of visceral sensitivity: from basic to clinical investigations. Gastroenterology 1997;112:1714-43.

8 Woodsworth RS, Sherrington CS. A pseudoaffective reflex and its spinal path. F Physiol (Lond) 1904:31:234-43.

9 Swain MG, Agro A, Blennerhassett P, et al. Increased levels of substance $\mathrm{P}$ in the myenteric plexus of Trichinellainfected rats. Gastroenterology 1992;102:1913-19.

10 Hwang L, Leichter R, Okamoto A, et al. Downregulation of neutral endopeptidase (EC 3.4.24.11) in the inflamed rat. Am F Physiol 1993;264:G735-43.

11 Vermillion DS, Collins SM. Increased responsiveness of jejunal longitudinal muscle in Trichinella-infected rats. Am jejunal longitudinal muscle
f Physiol 1988;254:G124-9.

12 De Giorgio R, Sternini C, Anderson K, et al. Tissue distribution and innervation pattern of peptide immunoreactivities in the rat pancreas. Peptides 1992;13:91-8.

13 De Giorgio R, Zittel TT, Parodi JE, et al. Peptide immunoreactivities in the ganglionated plexuses and nerve fibers innervating the human gallbladder. F Auton Nerv Syst 1995;51:37-47.

14 Vigna SR, Bowden JJ, McDonald DM, et al. Characterization of antibodies to the rat substance $\mathrm{P}(\mathrm{NK}-1)$ receptor and to a chimeric substance $\mathrm{P}$ receptor expressed in mammalian cells. 7 Neurosci 1994; 14:834-45.

15 Fan B-G, Wang L, Chen Y, et al. Pseudoaffective cardiac response to colonic distention in Sprague-Dawley rats. response to colonic distention
Gastroenterology 2000;118:A136.

16 Ho B, Wang L, Tougas G. The neurocardiac response to colonic distention is mediated through sympathovagal neucolonic distention is mediated through sympathovagal

17 Julia V, Su X, Bueno L, et al. Role of neurokinin 3 receptors on responses to colorectal distention in the rat: electrophysiological and behavioral studies. Gastroenterology 1999; 116:1124-31
18 Mayer EA, Raybould HE. Role of visceral afferent Mayer EA, Raybould HE. Role of visceral afferent mechanisms in function

19 Castagliuolo I, Keates AC, Qiu B, et al. Increased substance $\mathrm{P}$ responses in dorsal root ganglia and intestinal macrophages during Clostridium difficile toxin A enteritis in rats. Proc Natl Acad Sci USA 1997;94:4788-93.

20 Hurst SM, Stanisz AM, Sharkey KA, et al. Interleukin $1 \beta$-induced increase in substance $P$ in rat myenteric plexus. Gastroenterology 1993;105:1754-60.

21 Winston J, Shenoy M, Micci MA, et al. Tumor necrosis factor- $\alpha$ sensitizes primary neonatal sensory neurons in culture: the potential role of NF-kB in nociception. Gastroenterology 1999;116:A654.

22 Brown JL, Liu H, Maggio JE, et al. Morphological characterization of substance $\mathrm{P}$ receptor-immunoreactive neurons in the rat spinal cord and trigeminal nucleus caudalis. F Comp Neurol 1995;356:327-44.

23 Mantyh PW, Rogers SD, Honore P, et al. Inhibition of hyperalgesia by ablation of lamina I spinal neurons hyperalgesia by ablation of lamina I spinal neurons
expressing the substance P receptor. Science 1997;278:2759 .

24 Liu H, Brown JL, Jasmin L, et al. Synaptic relationship between substance $\mathrm{P}$ and the substance $\mathrm{P}$ receptor: light and electron microscopic characterization of the mismatch between neuropeptides and their receptors. Proc Natl Acad Sci USA 1994;91:1009-13.

25 McConalogue K, Bunnett NW. G protein-coupled receptors in gastrointestinal physiology II. Regulation of neuropeptide receptors in enteric neurons. Am F Physiol 1998;274:G792-6.

26 Abbadie C, Trafton J, Liu H, et al. Inflammation increases the distribution of dorsal horn neurons that internalize the neurokinin-1 receptor in response to noxious and nonnoxious stimulation. $\mathcal{F}$ Neurosci 1997;1 17:8049-60.

27 Marvizon JCG, Martinez V, Grady EF, et al. Neurokinin 1 receptor internalization in spinal cord slices induced by dorsal root stimulation is mediated by NMDA receptors. $\mathcal{F}$ Neurosci 1997;17:8129-36.

28 Abbadie C, Brown JL, Mantyh PW, et al. Spinal cord substance $P$ receptor immunoreactivity increases in both inflammatory and nerve injury models of persistent pain. inflammatory and nerve injury

29 Collins SM. The immunomodulation of enteric neuromuscular function: implications for motility and inflammatory disorders. Gastroenterology 1996;111:1683-99. 